\title{
François Eymard-Duvernay (dir.), Épreuves d'évaluation et chômage
}

Toulouse, Octarès, coll. « Le travail en débats », série « Entreprise, travail, emploi », 2012

\section{Gilles Raveaud}

\section{CpenEdition}

\section{Journals}

Édition électronique

URL : https://journals.openedition.org/travailemploi/6164

DOI : 10.4000/travailemploi.6164

ISSN : 1775-416X

Éditeur

DARES - Ministère du Travail

\section{Édition imprimée}

Date de publication : 14 décembre 2013

Pagination : 85-86

ISSN : 0224-4365

\section{Référence électronique}

Gilles Raveaud, «François Eymard-Duvernay (dir.),Épreuves d'évaluation et chômage », Travail et Emploi [En ligne], 136 | octobre-décembre 2013, mis en ligne le 01 octobre 2013, consulté le 03 août 2021. URL : http://journals.openedition.org/travailemploi/6164; DOI : https://doi.org/10.4000/travailemploi. 6164

Ce document a été généré automatiquement le 3 août 2021.

(c) Direction de l'animation de la recherche, des études et des statistiques (Dares) 


\section{François Eymard-Duvernay (dir.), Épreuves d'évaluation et chômage}

Toulouse, Octarès, coll. « Le travail en débats », série « Entreprise, travail, emploi », 2012

\section{Gilles Raveaud}

\section{RÉFÉRENCE}

François Eymard-Duvernay (dir.),Épreuves d'évaluation et chômage

, Toulouse, Octarès, coll. « Le travail en débats », série « Entreprise, travail, emploi »,

2012, 209 p.

\section{Une socio-économie politique du chômage}

L'ouvrage collectif dirigé par François Eymard-Duvernay défend l'idée selon laquelle le chômage est avant tout un phénomène d'ordre qualitatif. Au cœur de ce processus se trouvent des épreuves, multiples, auxquelles sont soumis les chômeurs. En fonction de leurs capacités, ils les franchissent avec plus ou moins de succès; de nature très variable, ces épreuves constituent autant d'étapes qui vont déterminer le devenir des

chômeurs sur le marché du travail, entre trajectoire professionnelle ascendante et exclusion durable de l'emploi.

L'ouvrage est constitué de cinq chapitres : le premier, consacré au « chômage d'exclusion » est théorique, les quatre autres traitent de questions empiriques. 
Dans son chapitre introductif, « Du chômage keynésien au chômage d'exclusion », François Eymard-Duvernay propose de « compléter » l'analyse keynésienne. Il ne s'agit pas seulement de savoir comment créer des emplois, mais de partir du fait que la compréhension de l'activité de travail renvoie à des dimensions cognitives (comment juger de la qualité du travail ?), éthiques (qu'est-ce qu'un « bon » travail ?), sociales

(comment les personnes se mettent-elles d'accord en situation de travail ?) et politiques (l'entreprise forme une communauté politique avec ses règles).

De ce fait, se mettent en place des « conventions » et des « régimes » de qualité, plus ou moins justes. Contre le « pouvoir autoritaire » des détenteurs de capitaux, François Eymard-Duvernay appelle à renforcer le pouvoir des personnes évaluées, par exemple en les associant à l'évaluation, ou en leur donnant la possibilité de contester celles qui leur ont été défavorables. Surtout, la croissance du produit intérieur brut (PIB) ne peut rien face au chômage d'exclusion, les régimes d'évaluation excluant les personnes pouvant perdurer une fois l'activité revenue. Il faut donc à tout prix restreindre la concurrence, « moyen privilégié de sélection », pour « développer des instances de débat dans lesquelles les conventions de qualité soient critiquées, remodelées en fonction des déséquilibres constatés » (p. 45).

Dans leur analyse de "La légitimité des épreuves de sélection », Guillemette de Larquier et Emmanuelle Marchal proposent une analyse statistique de l'enquête Offre d'emploi et recrutement (Ofer), menée en 2005 auprès des entreprises, et ayant recueilli plus de 4000 entretiens. L'objectif est de savoir si l'entreprise a rendu publique l'existence d'un poste à pourvoir, si elle a déposé son annonce auprès de Pôle emploi, etc.

$\mathrm{Au}$ total, notent les auteures, la plupart des établissements ont, au cours de leur recrutement, mobilisé au moins un critère discriminatoire, tels que l'état de santé, l'âge ou l'existence d'un handicap. De plus, elles relèvent que la présence de tels critères est d'autant plus fréquente que les qualifications demandées sont faibles.

Le traitement statistique des résultats permet de distinguer les procédures de recrutement « simplifiées » (un entretien tout au plus) des procédures « équipées » (plusieurs personnes impliquées dans le recrutement). Un second axe oppose les «jugements standards » (

curriculum vitae

[CV], lettre de motivation) aux jugements « contextuels ", lorsqu'un candidat est mis à l'épreuve dans une situation de travail concrète. Il apparait alors que les jugements standards sont défavorables aux primo-arrivants sur le marché du travail, quand les procédures simplifiées, ou contextuelles, réussissent davantage aux personnes peu diplômées.

L'analyse de «La recherche d'emploi » proposée par Emmanuelle Marchal et Delphine Remillon repose sur une analyse textuelle de 44 entretiens biographiques permettant aux auteures de mettre en évidence trois « mondes » du travail et du chômage.

Le premier est celui des « marchés internes » du travail. Il s'agit alors principalement de cadres, capables de présenter leur parcours comme une succession d'étapes cohérentes. Ce monde se distingue de celui des artisans qui travaillent dans de petits établissements, qui connaissent « une instabilité contractuelle » mais qui utilisent leur réputation pour passer d'un emploi à un autre. Le dernier monde est celui des personnes qui enchaînent des contrats de travail courts et instables, « sans cohérence 
Dans chaque cas, les instruments de recherche d'emploi et les attentes à l'égard des intermédiaires du marché du travail (écoles professionnelles, agences d'intérim, Pôle

etc.

) sont différents. Mais ce sont également les informations utilisées qui divergent : qualifications formelles, réputation, ou entrée dans un dispositif public. Surtout, notent Emmanuelle Marchal et Delphine Remillon, la «formalisation croissante » et «l'uniformisation » des méthodes de recrutement, avec par exemple la généralisation $\mathrm{du} \mathrm{CV}$ et de lettres de motivation « là où cela n'était pas nécessaire auparavant " (p. 104), accroissent le nombre d'épreuves, au détriment des moins bien dotés, tels que les chômeurs âgés. En réalité, comme elles le remarquent, « seuls les cadres semblent être en phase avec de telles exigences » (p. 105).

C'est ce que montre également l'analyse des « Généalogies du chômage » proposée par François Eymard-Duvernay et Delphine Remillon, et qui s'appuie sur 65 entretiens de type " parcours de vie ». Si ce chapitre a malencontreusement relégué les extraits des entretiens en annexe, on en appréciera le contenu ici retranscrit, qui souligne la violence et l'injustice que constitue le chômage, ce " parcours d'épreuves » marqué par

Enfin, Yolande Bennarrosh étudie le " point de vue des chômeurs » sur les institutions avec lesquelles ils sont en rapport, comme Pôle emploi. L'état de chômeur n'est en effet pas une donnée de la nature, mais une qualification sociale qui est fonction de l'image d'eux-mêmes que les institutions renvoient aux personnes privées d'emploi. Comme le pointe l'auteure, si les attitudes des chômeurs sont « en apparence similaires », au sens où ils répondent généralement aux injonctions qui leur sont adressées, cela n'empêche pas que leurs comportements divergent. Ainsi, les chômeurs les plus capables (au sens d'Amartya Sen) ont un rapport instrumental et détaché aux institutions, quand d'autres se mettent en retrait.

Et certains chômeurs se comportent par ailleurs de façon « militante » face à une institution qu'ils estiment illégitime, lui préférant « un système normatif opposé » (p. 192) à celui du service public de l'emploi, sous la forme des associations de chômeurs.

L'ouvrage a pour mérite de rappeler les fondations théoriques des recherches dont les résultats sont ici présentés, situées en économie mais aussi, et même principalement, en sociologie, avec notamment les travaux de Luc Boltanski. De même, il discute en profondeur les questions méthodologiques, notamment dans le traitement des entretiens ou à propos des analyses statistiques. Mais on aurait préféré que ces éléments soient présentés dans des chapitres séparés, afin de raccourcir des développements qui nous ont semblé trop longs et qui se répètent en partie d'un chapitre à l'autre. De plus, ce fort appareillage rend la lecture de l'ouvrage relativement ardue pour des lecteurs peu coutumiers de ces approches.

Surtout, nous avons pour notre part été frustré de l'excessive modestie des conclusions auxquelles parviennent les auteurs, qui ne sont pas à la hauteur de la prégnance du « chômage d'exclusion » et des violences infligées aux personnes injustement exclues 
L'appareillage théorique des auteurs est assumé comme étant normatif, et même « politique » : il s'agit de promouvoir une société juste, inclusive. Pourquoi alors ne pas avancer des propositions à la hauteur des problèmes si justement analysés ? Comme on le sait, les économistes néoclassiques ne se privent pas de faire des recommandations fortes et précises sur le niveau du Smic (salaire minimum interprofessionnel de croissance), la réforme des allocations chômage ou le droit du travail. Or ici, nous sommes en présence d'une analyse à la qualité scientifique bien supérieure. Certes, des

éléments de réforme sont mentionnés dans le livre, comme lorsqu'il est proposé de modifier les épreuves de recrutement par une prospection directe auprès des entreprises et par un accompagnement personnalisé du demandeur d'emploi (p. 145), ou encore d'interdire la photographie sur les CV (p. 63) - d'excellentes suggestions en effet. De même, la proposition de restreindre la concurrence sur le marché du travail est intéressante. Mais les auteurs ne sont pas suffisamment précis sur la possibilité de mise en œuvre de ces réformes.

Surtout, ces propositions ne sauraient être suffisantes face à l'ampleur du problème.

Faut-il interdire les licenciements ? Doit-on contrôler - et comment - les modes de recrutement ? Est-il souhaitable et possible d'assurer une garantie d'emploi par la puissance publique « en dernier ressort »? Une modulation des cotisations sociales versées par les entreprises selon les types de contrat de travail qu'elles utilisent constitue-t-elle une piste intéressante ? Sur tous ces points et bien d'autres encore, on attend des économistes des conventions qu'ils descendent dans l'arène, et qu'ils débattent avec les économistes libéraux, keynésiens, marxistes ou écologiques des solutions à apporter au problème du chômage, source de souffrances inouïes pour les

\section{AUTEURS}

\section{GILLES RAVEAUD}

Université Paris 8 - Institut d'études européennes 